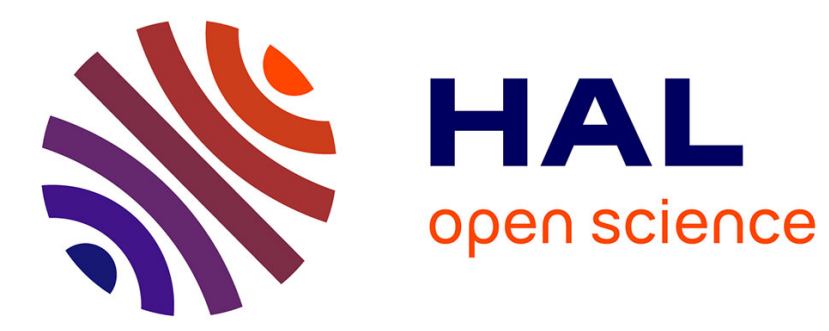

\title{
L'industrie cinématographique roumaine au service de la nation. Les enjeux de la production des films sur l'antiquité durant la période communiste
} Aurelia Vasile

\section{- To cite this version: \\ Aurelia Vasile. L'industrie cinématographique roumaine au service de la nation. Les enjeux de la production des films sur l'antiquité durant la période communiste. History of communism in Europe, 2012, 3/2012. halshs-01212772}

HAL Id: halshs-01212772

https://shs.hal.science/halshs-01212772

Submitted on 3 Jul 2017

HAL is a multi-disciplinary open access archive for the deposit and dissemination of scientific research documents, whether they are published or not. The documents may come from teaching and research institutions in France or abroad, or from public or private research centers.
L'archive ouverte pluridisciplinaire HAL, est destinée au dépôt et à la diffusion de documents scientifiques de niveau recherche, publiés ou non, émanant des établissements d'enseignement et de recherche français ou étrangers, des laboratoires publics ou privés.

$$
\text { Copyright }
$$




\section{L'industrie cinématographique roumaine au service de la nation. Les enjeux de la production des films sur l'antiquité durant la période communiste}

\section{Aurelia Vasile}

Résumé : L'objet d'analyse de cette étude est la production des films traitant l'antiquité et réalisés en Roumanie pendant le régime communiste. Il s'agit plus précisément de trois films : Les Daces (1965, Sergiu Nicolaescu), La Colonne (1968, Mircea Drăgan) et Burebista (1980, Gheorghe Vitanidis). Leur production témoigne des conditions politiques, idéologiques et économiques qui ont marqué le processus de reconstitution historique. Cet article tente de retracer le cheminement décisionnel dans la production de ces films, les objectifs des cinéastes et des autres professionnels du cinéma, le rôle du pouvoir politique dans leur évolution des années 1960 aux années 1970.

Mots-clés : politiques cinématographiques, coproductions, communisme, péplum, discours national

A l'image des autres démocraties populaires gravitant autour de l'Union Soviétique, la Roumanie connait un processus massif de nationalisation de toutes les branches de la production. Le cinéma n'y échappe pas et en 1948 a lieu la publication officielle de l'acte de nationalisation. Désormais, le film rejoint les autres arts et devient une préoccupation constante des autorités politiques qui commencent à accorder une importance grandissante à la production et à la diffusion de la culture dans un but éducatif clairement affirmé. Si au début des années 1950 le style réaliste-socialiste, imposé par le parti, limite fortement les possibilités individuelles d'expression artistiques ${ }^{1}$, après la mort de Staline, mais surtout à partir du début des années 1960, le film roumain témoigne d'une plus grande souplesse au niveau des thèmes et des approches esthétiques, une plus grande ouverture aux goûts du public. Cette évolution de nature culturelle trouve ses origines dans le rapport plus ou moins imbriqué entre le champ politique et le champ de la production culturelle et cinématographique en particulier.

\footnotetext{
${ }^{1}$ Les premiers films des années 1950 mettent en scène, dans le style réaliste-socialiste des héros de l'actualité : ouvriers, paysans ou ingénieurs. Angelo MITCHIEVICI, "Când viaţa învinge, răsună valea", In Paul CERNAT, Ion MANOLESCU, Angelo MITCHIEVICI, Ioan STANOMIR, Explorări în comunismul românesc, vol. 3, Bucureşti: Polirom, 2008.
} 


\section{Repères théoriques et méthodologiques}

Cette étude se propose d'examiner la place du film d'inspiration historique et en particulier du film traitant de l'antiquité dans le paysage cinématographique roumain, tout en essayant de voir les enjeux politiques et culturels de tel choix. Afin d'apporter des éléments de compréhension à cette problématique, nous avons choisi de retracer le cheminement de la production de trois films roumains sur l'antiquité (Les Daces (1965, Sergiu Nicolaescu), La Colonne (1968 Mircea Drăgan) et Burebista (1980, Gheorghe Vitanidis)) en analysant quelques éléments de nature politique, culturelle et sociale liés aux mécanismes de production. Ainsi, cet article se limitera à une analyse en amont des produits cinématographiques se focalisant sur la genèse des projets et l'enchevêtrement institutionnel de décision sans procéder à une analyse des films ni à une étude de la réception. Ces deux derniers thèmes doivent faire l'objet d'une analyse différente qui, en articulation avec la nôtre, permettrait la restitution plus complète du tableau politique et social de la représentation cinématographique de l'antiquité.

Cette étude se place au croisement des approches d'histoire culturelle et sociale qui s'inspire de la sociologie de l'art et de la production culturelle telle qu'elle est formulée par Howard Becker pour qui l'art, avant d'être une structure esthétique, représente un monde. Notre démarche tente, à l'exemple de Becker, d'appréhender "la complexité des réseaux coopératifs, dont l'art procède" ${ }^{2}$. Il s'agit, d'une part, de retracer de manière empirique les interactions administratives ou socioprofessionnelles du milieu cinématographique et d'autre part, de comprendre leur logique de fonctionnement, les intentions qui précèdent le résultat final. Cette approche purement "interactionniste" ${ }^{3}$ doit être placée dans le contexte des sociétés socialistes et en particulier celle du régime de Ceauşescu où la relation du parti et de l'Etat avec la production de film connaît des traits qui lui sont spécifiques. Malgré la centralisation excessive et la dépendance financière de l'industrie cinématographique des ressources fournies par l'Etat, les rapports entre les deux instances ne sont pas fondés sur la manipulation politique. Pour soutenir cette position, nous nous appuyons sur le système explicatif proposé par Katherine Verdery qui démontre la faiblesse de l'État, malgré ses multiples moyens de contrôle ${ }^{4}$. C'est pourquoi les concepts de politiques culturelles, compétition, négociation sont préférables à celui de propagande qui implique la soumission inconditionnelle de la culture au pouvoir. Nous utilisons également le terme plus ambigu "d'idéologie", mais il n'a pas ici la

\footnotetext{
${ }^{2}$ Howard S. BECKER, Art worlds, Berkley/Los Angeles/London: University of California Press, 1982, p. 1.

${ }^{3}$ Nathalie HEINICH, La sociologie de l'art, Paris: La Découverte, 2004, p.80.

${ }^{4}$ Katherine VERDERY, Compromis şi rezistenţă. Cultura română sub Ceauşescu, Bucureşti: Humanitas, 1994.
} 
connotation péjorative avec laquelle il a été investi dans la grande majorité des ouvrages sur les régimes communistes. Il n'est pas synonyme d'une représentation mensongère de la réalité qui s'opposerait à une quelconque vérité et ne fait pas référence de manière générale à l'idéologie communiste. Le mot est utilisé dans un sens plus restreint, et plus neutre qui renvoie à un ensemble d'idées et valeurs réunies sous une forme cohérente et qui produit une certaine forme d'adhésion.

Cette étude reposera sur un ensemble de documents inédits issus des Archives Nationales du Film, des Archives du Ministère de la Culture et des Archives du Parti Communiste Roumain. A cela s'ajoutent les enquêtes de production réalisées par la revue Cinema, quelques témoignages publiés après 1989 et l'entretien personnel avec le scénariste Mihnea Gheorghiu réalisé en 2005 à Bucarest.

\section{La production de film dans les années 1960 et l'apparition de l'épopée nationale}

Durant la deuxième moitié des années 1960 se produit un changement de paradigme idéologique et le discours de type marxiste pratiqué durant les années 1950 s'efface aux dépens de l'affirmation des valeurs nationales. Ce phénomène apparaît sur le fond de l'éloignement politique de la vision centraliste de Moscou, tendance qui culmine avec la déclaration d'indépendance du Parti Communiste Roumain en 1964. Suite à son élection comme leader du parti en 1965, Ceauşescu développe un discours de plus en plus prononcé en faveur de la nation qui est mise en accord avec l'histoire du mouvement communiste ${ }^{5}$.

Dans ce contexte, le monde du cinéma connaît une restructuration humaine et institutionnelle qui a marqué sensiblement le développement du film roumain. La nomination à la tête du Comité d'Etat pour Culture et Art (CSCA) en 1965 de l'architecte Pompiliu Macovei correspond à une approche plus mercantile du monde de la culture ${ }^{6}$. Jusqu'en 1968 il est secondé dans l'administration du cinéma par Mihnea Gheorghiu, grand promoteur de la rhétorique nationale, mais également supporter et défenseur des cinéastes moins orthodoxes ${ }^{7}$. Les restructurations de 1965 relèvent d'une approche plus professionnelle de l'administration de la culture qui est confiée à des fonctionnaires choisis sur des critères de compétence liées au domaine d'affectation. Les politiques culturelles et en particulier cinématographiques au

\footnotetext{
${ }^{5}$ Katherine VERDERY, op.cit., pp. 97-102.

${ }^{6}$ Anneli Ute GABANYI, Literatura şi politica în România după 1945, Bucureşti: Editura Fundaţiei culturale române, 2001, p. 140

${ }^{7}$ Le témoignage de Mircea Saucan vient appuyer cette idée. Iulia BLAGA, Fantasme şi adevăruri. O carte cu Mircea Săucan, Bucureşti: LiterNet, 2007, p. 80.
} 
cours des années 1960 se caractérisent par deux, sinon trois tendances placées souvent en compétition: exigences idéologiques, logique marketing et orientation artistique ${ }^{8}$.

Les films historiques des années 1960, surtout ceux réalisés en coproduction, étaient certainement le seul genre qui réussissait à réunir au moins deux de ces conditions (idéologique et commerciale) et selon certains critiques de film, ils témoignaient d'un achèvement artistique important ${ }^{9}$.

Du point de vue idéologique, le film d'inspiration historique produit dans les années 1960 se détache de plus en plus des sujets insurrectionnels ${ }^{10}$ proposés jusqu'alors et adopte peu à peu des thèmes nationaux. L'évolution est graduelle, du social au national ${ }^{11}$ et quelques films sont témoins de cette transition: Les Faucons (Neamul Şoimărestilor, Mircea Drăgan, 1964), Les Haïdouks (Haiducii, Dinu Cocea, 1965), mais surtout Tudor (Lucian Bratu, 1962). Ces films ont représenté l'avant-gout d'un projet cinématographique à part entière, devenu également un genre en soi : "l'épopée nationale cinématographique". Elle a été promue par Mihnea Gheorghiu au moment de son arrivée à la direction des différentes institutions cinématographiques (président du Conseil de la cinématographie entre 1963 et 1965 et viceprésident du Comité d'Etat pour Culture et Art entre 1966 et 1968) ${ }^{12}$. Il est soutenu par la nouvelle équipe politique installée à la tête du parti en 1965 qui apprécie et encourage cette idé $^{13}$. Le projet envisage une série de films censés reconstituer des moments précis et réels

\footnotetext{
${ }^{8}$ Aurelia VASILE, "La production cinématographique roumaine dans le labyrinthe étatique et social à la fin des années 1960 et au début des années 1970", In : Silvia MARTON, Anca OROVEANU, Florin ŢURCANU (eds.), L'Etat en France et en Roumanie aux XIXe et XXe siècles. Bucarest: New Europe College, 2011.

${ }^{9}$ Dans la chronique du film Les Daces, publiée dans la revue Cinema, Valerian Sava, apprécie : "[...] nous avons la révélation d'un univers thématique d'une inattendue force suggestive. Le film constitue pour notre cinéma une démonstration des possibilités. Les Daces placent notre film historique dans la perspective des grands et durables succès", In Cinema, février 1967, n², p. 9.

${ }^{10}$ Les films comme Lupeni '29 (Mircea Drăgan, 1962), L'Hiver en flammes (Răscoala, Mircea Mureşan, 1965), Golgotha (Mircea Drăgan, 1966)

${ }^{11}$ Le phénomène n'est pas spécifique au cinéma, mais se retrouve à d'autres niveau de la société: recherche scientifique, littérature, presse, discours politiques. Lucian BOIA, Istorie şi mit în conştiinţa românească, Bucuresti: Humanitas, 2002, p. 120.

${ }^{12}$ Mihnea Gheorghiu affirme avoir voulu, en tant que président du Conseil, que la cinématographie renoue avec «le fil naturel et traditionnel de la pensée des artistes sur leur nation » et «faire l'épopée nationale cinématographique » qui a été, dit-il, reprise par ses collègues. Archives personnelles, entretien avec Mihnea Gheorghiu réalisé en août 2005. L’initiative de Mihnea Gheorghiu dans la promotion de l'épopée est confirmée par le réalisateur Gheorghe Vitanidis dans le discours prononcé lors de la réunion de travail du 5 mars 1971 entre les cinéastes et Ceauşescu. Arhivele Naţionale Istorice Centrale, Bucureşti, ANIC (Les Archives Nationales Historiques Centrales, Bucarest), Fonds C.C. al P.C.R. Secţia Propagandă şi Agitaţie (Fond du Comité Central du Parti Communiste Roumain, Section Propagande et Agitation), dossier 2/1971, f. 73. Pourtant, d'autres personnes s'approprient l'idée. L'écrivain Eugen Barbu déclare en 1965, lors de la réunion du Conseil artistique du studio au sujet du film Les Daces, avoir salué la mise en œuvre de «l'épopée », pour laquelle il a milité depuis 10 ans. $C f$. ANF dossier "Dacii", "Stenograma şedinţei Consiliului Artistic ţinută în ziua de 6 septembrie 1965 ", p. 16.

${ }^{13}$ La Section "Littérature et Art" approuve cette proposition et demande un meilleur investissement des autorités culturelles pour sa promotion. ANIC, Fonds C.C. al P.C.R. Secţia Cancelarie (Fond du Comité Central du Parti
} 
du passé national roumain. Le sujet qui paraissait s'imposer de soi pour démarrer l'épopée fût la redécouverte des origines nationales, les Daces et les Romains. La perspective de consacrer à ce thème les ressources du studio enthousiasme et mobilise les fonctionnaires de la cinématographie qui veulent porter à l'écran des personnages importants pour l'histoire des Roumains $^{14}$.

$\mathrm{Du}$ point de vue économique, ce projet profite de l'ouverture des établissements cinématographiques roumains vers la collaboration avec des homologues capitalistes. C'est un phénomène qui se déroule sous les auspices du pouvoir politique qui initie les premiers projets de coopération culturelle avec des pays occidentaux ${ }^{15}$. Les coproductions cinématographiques, aux côtés des prestations de services ont représenté la forme la plus répandue de coopération internationale dans le milieu du film. Ce n'est pas une originalité du système roumain. Les entreprises occidentales saisissent la possibilité de l'ouverture idéologique et économique des pays socialistes et entreprennent des tournages à des prix plus avantageux que ceux pratiqués dans l'ouest. La Yougoslavie, la Bulgarie ${ }^{16}$, la RDA ${ }^{17}$, la Roumanie gagnent une réputation considérable sur le marché ouest européen pour ce type de services.

3. La production des péplums dans les années 1960 entre affirmation du nationalisme et synchronisation avec l'occident

\subsection{Les guerres daco-romaines: un projet pour un partenariat roumain- européen}

Initialement trois films différents ont été prévus pour suivre l'histoire des Daces et de leur dirigeant Decebal jusqu'à la conquête romaine et à la formation du peuple roumain ${ }^{18}$. Finalement, ce projet est réduit à deux films : Les Daces et La Colonne. La mise en scène du premier est confiée en 1966 à un réalisateur débutant, qui sera consacré, par la suite, comme le spécialiste des superproductions en Roumanie, Sergiu Nicolaescu. Le deuxième est réalisé

Communiste Roumain, Section Chancellerie), dossier 67/1966, "Referat privind activitatea Centrului de Producţie Cinematografică Buftea în perioada 1960-1965", f. 51.

${ }^{14}$ Pour une analyse détaillée de la production des films historiques roumains voir Aurelia VASILE, Le cinéma roumain dans la période communiste. Représentations de l'histoire nationale, Bucureşti: Editura Universităţii din Bucureşti, 2011.

${ }^{15}$ Parmi les pays ciblés par le gouvernement se trouvent la France, la Belgique, le Danemark, la Norvège. AMC, dossier 20845/1966, «Planuri şi acorduri culturale : 1965-1966 », lettre du 23 novembre 1964 du Ministère des Affaires Etrangères adressée à Constanţa Crăciun, présidente du CSCA.

${ }^{16}$ Mira et Antonin LIEHM, Les Cinémas de l'Est, Paris: les éditions CERF, 1989, p. 333.

${ }^{17}$ La RDA développe ce type de collaboration presque à la même période que la Roumanie, dans la deuxième moitié des années 1950. Cyril BUFFET, Défunte DEFA. Histoire de l'autre cinéma allemand, Paris: les éditions du CERF, 2007, pp. 116-121.

18 Arhivele Naţionale de Film, Bucureşti, ANF (Les Archives Nationales de Films), dossier "Dacii", "Stenograma şedinţei Consiliului Artistic ţinută în ziua de 6 septembrie 1965", p. 19. 
en 1968 par un metteur en scène avec une expérience accumulée dans des films à thématique historique, Mircea Drăgan. Les deux films ont porté la signature du scénariste Titus Popovici. Le premier scénario a été probablement écrit durant l'année 1964, car en septembre 1965 il est débattu par le Conseil artistique du studio et les premiers essais avec des acteurs roumains sont réalisés par Sergiu Nicolaescu à la fin de l'été de $1965^{19}$.

Fortes de l'expérience acquise suite aux prestations de services pour des maisons de production étrangères ${ }^{20}$ et aux coproductions de la fin des années 1950 , début du $1960^{21}$, les institutions cinématographiques roumaines engagent des négociations pour le co-financement des films sur l'histoire des Daces. Les discussions avec la maison de production française Franco-London Film ${ }^{22}$ se déroulent tout au long de l'année 1965. D'ailleurs, vers 1964, la compagnie française "croulait sous les dettes"23 et c'est probablement à ce moment-là qu'elle se tourne vers les coproductions avec les pays socialistes. En ce qui concerne Les Daces, il est clair qu'elle cherche à financer des productions à grand succès d'après le modèle des péplums franco-italiens de la fin des années 1950 et du début des années 1960. Nous pouvons même considérer ces productions roumaines comme le dernier souffle du deuxième "âge d'or" du péplum ${ }^{24}$.

Les contacts noués au cours des années 1965 et 1966 se finalisent par un accord de principe, signé en mars 1966 entre la France et la Roumanie, valable pour deux ans, avec des prérogatives générales sur la coproduction et sur l'échange de films ${ }^{25}$. Le contrat pour le film Les Daces prévoit pour la partie roumaine une participation de $60 \%$, tandis que les représentants occidentaux imposent la distribution des rôles principaux ${ }^{26}$.

\footnotetext{
${ }^{19}$ Dan ARNĂUTU, "Dacii la Buftea. Şantier", In Cinema, novembre 1965, n 11, p.10.

${ }^{20}$ Les Fêtes galantes (Serbările galante, René Clair, 1965) et Sept gars et une garce (Şapte băieţi şi o ştrengăriţă, Bernard Borderie, 1966), Pour la conquête de Rome (Bătălia pentru Roma, Robert Siodmak, 1968).

${ }^{21}$ La Citadelle écroulée (Citadela Sfărâmată, Marc Maurette, 1957) et Les Chardons du Baragan (Ciulinii Bărăganului, Louis Daquin, 1958), Codine (Henri Colpi, 1963).

${ }^{22}$ Le directeur de la société était Henry Deutschmeister (1903-1969), un producteur d'origine juive né en Roumanie, probablement à Brăila. Il fuit la montée du fascisme et l'expansion allemande pour se réfugier en France. Ulli PICKARDT, Travelling arrière: mémoires d'un cinéaste, Paris: l'Harmattan, 2004, p. 138. D'ailleurs, il fait partie d'une génération de producteurs et de réalisateurs immigrants d'Union Soviétique ou d'Europe Centrale, poussée vers Paris pour les mêmes raisons durant les années 1930 (Colin CRISP, The Classic French cinema, 1930-1960, Bloomington: Indiana University Press, pp. 168-170.) Le lieu de développement de leurs sociétés était les Champs-Elysées. Marcel l'Herbier note dans ses mémoires, non sans irritation, la multiplication de leurs compagnies et les scripts d'inspiration communiste apportés de Russie (Marcel L'HERBIER La tête qui tourne cité par Colin CRISP, op.cit., p. 170.)

${ }^{23}$ Ulli PICKARDT, op. cit., p. 138.

${ }^{24}$ Michel ÉLOY « Rome », In CinémAction. Le Péplum : l’Antiquité au cinéma, n 89, 1998, p. 57.

${ }^{25}$ ANIC, Fonds C.C. al P.C.R. Secţia Cancelarie, dossier 18/1966, "Angajament cinematografic de coproducţie şi schimb de filme între RSR şi Republica Franceză. Proiect", ff. 26-30.

${ }^{26}$ Bujor T. RIPEANU, Filmat în România Un repertoriu filmografic, vol.1, Bucureşti: Fundaţia Pro, 2004, p. 139.
} 
On a affaire à deux logiques différentes : celle des producteurs étrangers et celle du studio roumain. D'une part, les Occidentaux cherchent à réaliser des films selon le modèle des péplums classiques en exigeant des acteurs connus pour ce genre de rôles ${ }^{27}$ : Pierre Brice, George Marshall, Marie José Nat pour Les Daces et Richard Johnson, Amedeo Nazzari et Antonella Lualdi pour La Colonne. D'autre part, l'équipe de réalisateurs, écrivains et autres professionnels roumains, convoquée pour débattre du scénario ${ }^{28}$, oscille entre le désir d'aligner ce film sur les productions occidentales du même genre et le souci de conférer une image riche et rigoureusement documentée aux Daces en explorant l'univers politique et social de l'époque ${ }^{29}$. La stéréotypie esthétique et dramatique spécifique au péplum ${ }^{30}$ exclut les exigences documentaires de l'approche éducative.

Les membres du studio roumain sont spectateurs et admirateurs des superproductions européennes et hollywoodiennes sur l'antiquité. L'un des films occidentaux vu comme modèle, dans son genre, par la majorité des intervenants est Les Vikings (Richard Fleischer, 1958). Le rédacteur Petre Sălcudeanu, le directeur de la photographie Aurel Samson et le réalisateur Ion Popescu-Gopo considèrent Les Vikings comme l'un des meilleurs films du genre parce qu'il met en scène une confrontation entre des caractères très puissants ${ }^{31}$. C'est pourquoi ils conseillent au scénariste de réduire les amples descriptions de la vie politique dace et d'organiser le récit, selon le modèle américain, autour de deux personnages masculins : Decebal et Severus. Toutefois, la perspective de produire un péplum autochtone et d'abandonner la réflexion historique pour se plier aux codes spécifiques du genre, détermine la majorité des participants aux discussions à contrebalancer le poids des éléments spectaculaires par l'exigence d'une rigueur scientifique.

A la différence de nombreux péplums des années 1950-1960 où l'antiquité n'est qu'un cadre exotique et éloigné, prétexte pour mettre en scène des personnages et des actions héroïques ${ }^{32}$, ces films accordent une attention particulière aux sources historiques. Le film bénéficie des conseils d'un historien professionnel, l'un des spécialistes de la question, Constantin Daicoviciu. La section de propagande du parti atteste sa contribution et précise qu'il "a

\footnotetext{
${ }^{27}$ Laurent AKHNIN, « Dicopéplum », CinémAction. Le Péplum : l'Antiquité au cinéma, n 89, 1998, pp. 100125.

${ }^{28}$ Le 6 septembre 1965 a lieu une réunion du Conseil Artistique du studio "Bucureşti" afin de discuter les premières phases d'écriture du scénario Les Daces.

${ }^{29}$ Les discussions qui ont lieu au sein du Conseil Artistique au sujet du scénario laissent entendre que la première version était axée sur les aspects politiques de la vie des Daces, ce qui disparaît de la version finale. ANF dossier "Dacii", "Stenograma şedinţei Consiliului Artistic ţinută în ziua de 6 septembrie 1965", pp. 2-5.

${ }^{30}$ Pour une analyse des stéréotypes des péplums voir Frédéric MARTIN, L'Antiquité au cinéma, Paris: Dreamland, 2002.

${ }^{31}$ ANF dossier "Dacii", "Stenograma şedinţei Consiliului Artistic ţinută în ziua de 6 septembrie 1965", p. 5.

${ }^{32}$ Pierre SORLIN, Film in History: Restaging the Past, Oxford: Blackwell Publishers, 1980.
} 
apprécié la valeur du texte littéraire et a proposé des modifications au sujet des coutumes, de la dénomination et de l'âge de certains personnages" ${ }^{33}$. Selon la même note, les modifications ont été opérées et l'historien restera près de l'équipe de tournage durant la réalisation du film. La reconstitution des éléments figuratifs (costumes, décors, armes, accessoires) fait partie des objectifs importants des auteurs. Nicolaescu affirme que la source d'inspiration a été la Colonne de Trajan et le monument d'Adamclisi ${ }^{34}$. Ileana Oroveanu, la réalisatrice des costumes du film La Colonne, témoigne, en effet, d'avoir pris de nombreuses photos de la Colonne de Trajan qui a constitué la base de la création ${ }^{35}$. Certes, la manière dont ces sources sont interprétées et transposées à l'écran est discutable ${ }^{36}$, mais on remarque un effort indéniable de recherche d'authenticité.

Fort de l'expérience acquise lors de la production de la première partie de l'histoire des Daces, le studio démarre la deuxième partie, à savoir la conquête de la Dacie et le processus d'ethnogenèse. Le film La Colonne est également une coproduction, réalisée cette fois-ci avec une compagnie ouest-allemande, la Central Cinema Company Filmkunst ${ }^{37}$. Comme dans le cas du film Les Daces, la participation financière est majoritairement roumaine. Le studio "Bucureşti" contribue avec 21 millions de lei, le CCC Filmkunst avec un million de marks allemands, apporte la pellicule, choisit et rémunère les interprètes principaux et se charge de l'exploitation internationale ${ }^{38}$. A l'instar de Franco-London Film, l'intérêt de l'entreprise allemande est surtout commercial, raison pour laquelle ses représentants exigent la réécriture du scénario afin d'élargir le rôle des trois vedettes internationales ${ }^{39}$ et pour la diffusion occidentale, l'allongement des scènes de combat ${ }^{40}$. Comme auparavant, les producteurs roumains ont cherché un équilibre entre ces exigences de nature commerciale et leurs propres considérations sur le passé national.

\footnotetext{
33 ANIC, Fonds C.C. al P.C.R. Secţia Propagandă şi Agitaţie, dossier 31/1966, "Dacii", f. 49.

34 "Panoramic peste platouri : Mihai Viteazul - un nou film românesc de Sergiu Nicolaescu", Cinema, n $^{\circ} 3$, mars 1968.

35 "Panoramic peste platouri : Zărneşti secolul XX. Dacia Secolul II", Cinema, n 1, janvier 1968, p. 28.

36 A ce sujet, voir la partie consacrée à la mise en parallèle des sources et de leur transposition cinématographique dans Oscar Lapeña Marchena "Nuevas perspectivas en la recepción cinematográfica de la historia antigua: el ejemplo de Dacii/Les guerriers (Sergiu Nicolaescu, Rumania/Francia 1966)", Studia historica. Historia antigua, $\mathrm{n}^{\circ} 28,2010$, pp. 155-178.

${ }^{37}$ ANIC, Fonds C.C. al P.C.R. Secţia Cancelarie, dossier 88/1968, "Proiectul planului tematic de perspectivă", f. 210.

${ }^{38}$ Ibidem

${ }^{39}$ AMC, carton CSCA Dispoziţii vol.XVII, 1968, pp. 12-13.

${ }^{40}$ Bujor T. RIPEANU, op.cit., vol. 1, p. 139.
} 


\subsection{Quelle image façonner aux Daces? Choix pour une reconstitution}

Les préparatifs qui ont conduit à la réalisation des films sur les Daces ont mis en relief deux préoccupations majeures concernant la création du monde dace. Elles vont de pair. La première a été de mettre en place un ensemble d'éléments qui désignent les Daces comme les ancêtres des Roumains, la deuxième a insisté sur le soin spécial de l'image des Daces en vue de leur sortie officielle sur les écrans européens comme personnages de cinéma.

Il y a un esprit nationaliste dans la façon d'approcher le sujet, comparable probablement, à un certain niveau, avec les mises en scène françaises des Gaulois ${ }^{41}$. De manière évidente tous les participants au film voient dans les Daces les véritables ancêtres des Roumains ${ }^{42}$. Certes, l'élément romain n'est pas négligé. Les participants au débat sur le scénario Les Daces, ainsi que les participants à la production du film La Colonne cherchent à mettre en valeur les qualités des Romains, mais au final le portrait des Daces semble les concerner davantage. Leurs costumes constituent pour Sergiu Nicolaescu l'un des éléments qui prouvent l'identification des Daces au peuple roumain contemporain car il est convaincu que "lorsque nous avons habillé les figurants dans ces costumes, nous nous sommes retrouvés devant un groupe de paysans tels que nous pouvons rencontrer aujourd'hui dans Oaş ou dans d'autres régions du pays" ${ }^{43}$. Ileana Oroveanu, l'auteur des costumes pour le film La Colonne fait des appréciations similaires : "en examinant les portraits de la Colonne de Trajan, j'ai eu tout le temps la surprise de trouver des éléments vestimentaires qui se sont perpétués jusqu'à nos jours. Les chaussures avec pointe se portent même aujourd'hui dans la région de Cărbunari, les pantalons longs, la chemise en lin se portait alors longue et avec des manches longues"44. De plus, les acteurs roumains prennent la mesure de leur personnage en faisant ressortir les similitudes entre les deux peuples. L'acteur Amza Pellea considère son personnage Decebal (commun aux deux films) comme "le prototype des meilleurs traits de notre peuple qui ont traversé les siècles et qui vont faire partie de l'esprit national roumain pour toujours [...] Il représente l'essence de notre esprit national sur lequel la superposition romaine a généré des combinaisons heureuses et a accentué les qualités des Daces jusqu'à la brillance" ${ }^{45}$. De même, l'acteur Ştefan Ciubotăraşu, en référence à son personnage, le dace Ciungu, témoigne vouloir "l'amener au dénominateur commun avec les traits de notre peuple, à savoir la bonté,

\footnotetext{
${ }^{41}$ Krzysztof POMIAN, "Francs et Gaulois", In Pierre NORA (eds.) Les Lieux de mémoires, vol. III, Les France, Paris: Gallimard, 1997, pp. 41-105.

${ }^{42}$ On reconnaît dans cette tendance, le penchant pour la résurrection des origines autochtones les plus éloignées spécifiques d'un certain courant nationaliste formé après 1900. Lucian BOIA, op.cit., p. 98.

43 "Panoramic peste platouri : Mihai Viteazul - un nou film românesc de Sergiu Nicolaescu", entretien avec Sergiu Nicolaescu, Cinema, mars 1968, $\mathrm{n}^{\circ} 3$.

44 "Panoramic peste platouri : Zărneşti secolul XX. Dacia Secolul II", Cinema, janvier 1968, n 1, p. 28.

45 "Panoramic peste platouri : cu Decebal despre Decebal", Cinema, août 1966, nº 8, p. 7.
} 
l'hospitalité, le bon sens et une grande expérience filtrée, à travers les siècles, par les larmes" 46 .

Le projet cinématographique autour des Daces rassemble de nombreux acteurs de la vie culturelle roumaine: historiens, écrivains, cinéastes, journalistes, comédiens et autres professionnels du cinéma. Réuni dans le conseil artistique du studio, chacun exprime un avis particulier sur ce peuple et sur la manière de le mettre en scène. Le responsable des décors aux deux films, Liviu Popa, est conscient de «la pauvreté des sources» et de ses conséquences sur la reconstitution, mais il déclare convaincu :

Il est connu que les Daces étaient très riches, ils avaient de l'or. L'or appartenait à l'Etat et l'Etat était Decebal [...] Tout ce qu'il y avait de meilleur dans le monde à ce moment-là se trouvait chez les Daces [...] Il est connu que les Daces possédaient un bon goût légendaire. Les formes primitives de leur architecture étaient très raffinées. Rien de mauvais goût. Rien surchargé, tout était fonctionnel, beau et utile $[\ldots]^{47}$.

De même, au sujet des problèmes de reconstitutions des décors pour le film La Colonne, Liviu Popa, témoigne avoir été mis dans la situation de "faire un grand effort d'imagination, de «fantaisier » de manière réaliste à partir des peu nombreux documents historiques" ${ }^{48}$. Le manque de sources est remplacé par un autre type d'inspiration: "l'inimaginable héritage laissé par les ancêtres" ${ }^{49}$, plus précisément par "leur formidable force de création, prouvée pleinement au cours des deux millénaires, héritage que nous aimerions faire connaître au monde entier" 50 .

Les mots de Popa donnent la mesure du véritable enjeu de la production de ces films: il ne s'agit pas de réaliser deux péplums supplémentaires, mais de faire découvrir au monde entier la place des Roumains dans l'histoire européenne. Ainsi, la préoccupation d'associer le monde dace aux sociétés traditionnelles roumaines est doublée par un souci d'idéalisation en vue de la diffusion internationale du film et de l'apparition pour la première fois sur la scène du cinéma mondial des Daces. Le réalisateur Ion Popescu-Gopo résume la situation :

Ce qui est nouveau dans ce film, ce sont les Daces. Les Romains nous les avons vus, les Mongols nous les avons vus ; pour les Daces ça va être la première apparition et il est très important de savoir comment ils vont se comporter. Je pense aux Daces comme à des demi-dieux. Qu'ils ne soient pas conçus comme les haïdouks [allusion au film d'aventures Les Haïdouks, Dinu Cocea, 1965 N.D.A.] ! Le scénario est bon, mais si vous ne faites pas attention à ce détail, ça va être laid et de mauvais goût [...] Il est nécessaire de réaliser ce genre de film, que nous attendons tous avec passion et impatience, mais je recommande de faire de ces Daces des idoles et non pas comme pour Les Haïdouks, où on a mis

\footnotetext{
46 "Panoramic peste platouri : Zărneşti secolul XX. Dacia Secolul II", In Cinema, janvier 1968, n 1, p. 27.

47 "Panoramic peste platouri : Anul 87 e.n. : Dacii", In Cinema, juillet 1966, n 7, p. 5.

48 "Panoramic peste platouri : Zărneşti secolul XX. Dacia Secolul II", Cinema, janvier 1968, n 1, p. 28.

${ }^{49}$ Ibidem.

${ }^{50}$ Ibid.
} 
quelques mèches de cheveux et des moustaches et voila tout. Je dis tout cela pour apprendre des erreurs passées $^{51}$.

L'écrivain Eugen Barbu exprime un avis similaire bien que dépourvu de la dimension mythologique évoquée par Gopo: "Nous devons apparaître sur le marché avec quelques choses de sérieux. Il ne faut pas faire de nos ancêtres des dieux, mais les données historiques existent et nous devons montrer de l'estime envers eux" ${ }^{52}$. Dans le même but, Petre Sălcudeanu va jusqu'à solliciter l'introduction des éléments originaux dans les coutumes des Daces, même si elles ne sont pas attestées historiquement, uniquement pour ne pas répéter des scènes déjà vues dans d'autres films comme Les Cosaques ou Les Vikings ${ }^{53}$.

La réussite de ces intentions est à apprécier en regardant le résultat final, mais également en analysant la réception des films, ce qui doit faire l'objet d'une future étude. En revanche, nous devons souligner l'apport de ces films pour le développement d'une expérience internationale sans précédent dans le milieu du cinéma roumain. Le Studio "Bucureşti" accueille de nombreuses personnalités internationales et devient l'une des destinations européennes importantes pour la réalisation des superproductions. De ce pont de vue, l'industrie cinématographique a été d'une certaine façon un lieu d'affirmation nationale.

En ce qui concerne les préparations et les négociations qui accompagnent la mise en production, celles-ci se déroulent en grande partie au niveau du studio. Bien que lindustrie cinématographique soit dépendante des plus hautes autorités politiques, l'immixtion des responsables politiques dans les questions de production est minimale. Comme nous l'avons vu, les opinions sur l'évolution artistique du film Les Daces appartiennent aux professionnels du cinéma. Certes, les validations financières et les autorisations thématiques se décident au niveau du ministère (le CSCA), mais les professionnels du studio semblent bénéficier d'une certaine autonomie dans leurs prises de décisions.

\section{La démesure nationaliste des années 1970 et le fleurissement d'un sujet inattendu : Burebista}

L'une des caractéristiques essentielles du milieu culturel des années 1970 est l'intensité du discours national qui se radicalise visiblement surtout dans la deuxième moitié de la décennie. C'est un phénomène qui se manifeste sur le fond d'une crise économique grandissante et d'un culte de la personnalité puisant ses repères dans les héros du passé $e^{54}$. Probablement le discours sur les origines nationales, accompagné par des interprétations

\footnotetext{
${ }^{51}$ ANF dossier "Dacii", "Stenograma şedinţei Consiliului Artistic ţinută în ziua de 6 septembrie 1965", p. 13.

${ }^{52}$ Ibidem, p. 17.

${ }^{53}$ Ibid., p. 6.

${ }^{54}$ Adrian CIOROIANU, Ce Ceauşescu qui hante les Roumains Bucureşti: Curtea Veche, l'AUF, 2004
} 
protochronistes, a connu les formes les plus extrêmes. La "dacomanie" (l'exaltation de l'élément dace dans l'ethnogenèse roumaine) et par la "thracomanie" (révélation de l'élément encore plus ancien, thrace) représentent les deux mythes indigénistes censés valoriser non seulement l'ancienneté des Roumains, mais aussi la supériorité de ces peuples antiques sur les autres civilisations de leur temps. C'est une forme de nationalisme révélé déjà comme doctrine fondatrice par la Garde de Fer, le mouvement d'extrême droite de l'entre-deuxguerres 55 .

Les premiers écrits d'inspirations thraco-dacomanes de l'époque communiste apparaissent vers 1975-1976, mais ce n'est qu'en 1977 que l'idée est adoptée sous une forme officielle par le parti. Le plénum du C.C. du P.C.R. du 26-27 octobre 1977 décide de célébrer en 1980 l'anniversaire des 2050 ans depuis la création du "premier État dace centralisé et indépendant" du roi Burebista. Cette déclaration déclenche un ample programme de manifestations culturelles et scientifiques mises en place par le CCES (le Conseil de la Culture et de l'Education Socialiste) destinées à célébrer l'évènement ${ }^{56}$. Depuis le début des années 1970, lorsque la logique des anniversaires prend le dessus dans le choix des sujets, la cinématographie a respecté avec fidélité les plans thématiques et en 1977 le plan thématique prévoit la réalisation d'un film de fiction sur le roi Burebista.

Le scénariste du film, Mihnea Gheorghiu, personnalité clé du cinéma roumain dans les années 1960, devient en 1972 président de l'Académie des Sciences Sociales et Politiques. Il assiste aux débats autour des Thraces et soutient les idées thracomanes portées par les historiens rattachés à l'institution qu'il présidait. Il se rappelle, lors d'un entretien qu'il nous a accordé ${ }^{57}$, les controverses autour de la question des Thraces, l'opposition qu'avait rencontrée l'idée et la force avec laquelle il avait appuyé le projet. L'Académie d'Etudes Sociales et Politiques publie en 1976 un volume, Thraco-Dacica, qui prône la descendance thrace des Roumains. Plus encore, Mihnea Gheorghiu accompagne l'ouvrage d'une préface dans laquelle il affirme que même le processus d'anthropogenèse aurait commencé sur le territoire de la Roumanie ${ }^{58}$.

\footnotetext{
${ }^{55}$ Florin CONSTANTINIU, De la Răutu şi Roller la Muşat şi Ardeleanu, Bucureşti: Editura Enciclopedică, 2007, pp. 388-391.

${ }^{56}$ AMC dossier 21162/1977, "Măsuri pentru aplicarea sarcinilor ce revin CCES din planul aprobat de Plenara CC al PCR din 26-27 octombrie privind aniversarea a 2050 de ani de la crearea primului stat dac, centralizat şi independent", pp. 26-27.

${ }^{57}$ Archives personnelles, entretien avec Mihnea Gheorghiu réalisé en août 2005.

${ }^{58}$ Vlad GEORGESCU, Politică şi istorie. Cazul comuniştilor români (1944-1977), Bucureşti: Humanitas, 1991, p. 99 .
} 
Dans ce contexte, Mihnea Gheorghiu écrit le scénario du film Burebista, probablement à la fin $1975^{59}$. D'ailleurs, son scénario n'est pas unique. Radu Theodoru propose également un scénario pour la télévision dès 1975 , intitulé Burebista ${ }^{60}$. A ce moment-là, le parti ne s'était pas encore emparé du personnage et Burebista n'était qu'un objet d'études historiques. C'est pourquoi, une fois le scénario cinématographique fini, la rédaction l'accepte, mais annonce l'impossibilité de procéder à l'entrée en production faute de financement ${ }^{61}$. D'ailleurs le sujet n'est intégré dans aucun plan thématique, de 1975 ou de 1976. Les directives du parti à la fin de 1977 changent la donne. La réalisation de ce film est un cas symptomatique des rapports entre les cinéastes, les bureaucrates et le parti. Cette pellicule est le lieu de convergence du centralisme du régime et de la libre initiative des auteurs. Il est évident que l'intérêt du parti pour Burebista et la prise en charge du projet par le CCES ont obligé le studio à démarrer la production du film. Mais il est tout aussi important de remarquer que le scénario était finalisé et proposé aux maisons de production probablement deux ans auparavant. Certes le financement lui a été refusé au départ et ce n'est qu'en 1977 que les fonds sont débloqués, mais l'idée avait fait surface dès 1975 sans être le résultat d'une commande officielle.

\subsection{Obstacles financiers et administratifs}

L'entrée en production est prévue pour $1978^{62}$ et la sortie pour juillet-août 1980 avec l'objectif de le présenter au Congrès mondial d'histoire ${ }^{63}$. A partir d'octobre 1977, le film Burebista devient une priorité pour la cinématographie roumaine. Les autres personnalités historiques prévues dans les plans thématiques, comme Mircea l'Ancien, Constantin Brâncoveanu et Petru Rareş sont redirigées vers le studio de films documentaires "Sahia", en attendant "des conditions matérielles appropriées" ${ }^{64}$ pour la réalisation de fictions.

\subsubsection{Le financement}

La réalisation du film est confiée dans une première phase à la maison de production $\mathrm{n}^{\circ} 4$, dirigée par Corneliu Leu. Bien que le film soit une commande du parti, la question du

\footnotetext{
${ }^{59}$ Le travail d'écriture du scénario est annoncé par le directeur de la Maison de production n ${ }^{\circ} 3$, Corneliu Leu. "Sub semnul epopeii naţionale", Cinema, n² 2, février 1976, p. 3.

${ }^{60}$ Dinu C. Giurescu réalise un compte rendu de spécialité au sujet du scénario en décembre 1975. Archives personnelles de Dinu C. Giurescu, professeur universitaire consultant, Faculté d'histoire, Université de Bucarest, et membre titulaire de l'Académie roumaine, "Referat", 22 décembre 1975.

${ }_{61}$ Archives personnelles, entretien avec Mihnea Gheorghiu réalisé en août 2005.

62 Arhivele Ministerului Culturii, Bucureşti AMC (Les Archives du Ministère de la Culture, Bucarest), dossier 21162/1977, "Măsuri pentru aplicarea sarcinilorce revin CCES din planul aprobat de Plenara CC al PCR din 2627 octombrie privind aniversarea a 2050 de ani de la crearea primului stat dac, centralizat şi independent", p. 26. 63 ANF, dossier "Burebista", lettre adressée par Dumitru Fernoagă à la Centrale "Romaniafilm", 21 janvier 1979.

${ }^{64}$ Ibidem.
} 
financement est problématique car Burebista s'annonce comme une superproduction et la cinématographie roumaine n'est pas en mesure d'en supporter le coût. Leu annonce un devis d'environ 35 à 40 millions de $1 \mathrm{ei}^{65}$, somme qui parât exorbitante pour le capital de la cinématographie roumaine à la fin des années 1970, surtout qu'après Michel le Brave aucune autre production n'avait atteint un tel niveau d'investissements. Les échanges et les négociations qui ont lieu entre la maison de production et la Centrale "Româniafilm", l'institution qui gère la distribution des ressources, témoignent des tensions entre les directeurs des deux établissements et des pressions économiques et idéologiques auxquelles elles étaient confrontées. Après les premiers refus de budget, Leu revoit les sommes à la baisse (24-30 millions de lei) ${ }^{66}$, mais la Centrale estime ce montant toujours irréalisable. D'un côté, le directeur de la maison de production trahit son impatience et suggère à la Centrale de faire appel au soutien de la télévision. De l'autre côté, la Centrale se montre très critique envers ces sollicitations empressées et met en cause la manière de travailler du directeur de la maison de production ${ }^{67}$. Le verdict financier établi par la Centrale est très sévère : 7 millions pour un film d'une série et 14 millions pour un film de deux séries ${ }^{68}$. Ces sommes représentent le montant habituel pour des "films de grande complexité" à la fin des années $1970^{69}$, mais elles ne garantissent nullement la qualité de reconstitution que le parti attendait pour l'événement.

La situation paraît sans issue, mais une solution salvatrice se profile au début de l'année 1979: l'alternative d'une co-production internationale. Corneliu Leu profite de la présence en Roumanie de l'homme d'affaires roumain installé en Italie, Iosif Constantin Drăgan ${ }^{70}$, pour approcher les studios italiens en vue d'un péplum. Afin de convaincre les Italiens, le sujet proposé n'est plus Burebista mais Burebista et César. La société contactée par Drăgan, Clesi Cinematografica, décline la proposition invoquant "la crise politique italienne" et "une décision judiciaire ayant mis en danger toute la cinématographie italienne, qui doit verser des

\footnotetext{
${ }^{65}$ ANF, dossier "Burebista", lettre adressée par Corneliu Leu à la Centrale "Româniafilm", 19 septembre 1978.

${ }^{66} \mathrm{ANF}$, dossier "Burebista", lettre adressée par Corneliu Leu à la Centrale "Româniafilm", 16 janvier 1979.

67 ANF, dossier "Burebista", lettre adressée par Alexandru Marin, directeur de la Direction technique et de production de la Centrale "Româniafilm" à Corneliu Leu, 2 février 1979. ${ }^{68}$ Ibidem.

${ }^{69}$ AMC, dossier 26077/1979,"Raport privind rezultatele economico-financiare ale sectorului cinematografic", p.11.

${ }^{70}$ Iosif Constantin Drăgan a été attiré par l'idéologie fasciste de la Garde de Fer pendant l'entre-deux-guerres et a continué à promouvoir les valeurs nationalistes roumaines. Il a eu des préoccupations semi-culturelles, a entretenu des relations avec les promoteurs du protochronisme roumain et exalté le rôle des Thraces dans l'histoire européenne en publiant un livre, Noi tracii şi istoria noastră multimilenară (Nous, les Thraces et notre histoire multimillénaire), publié en français sous le titre Les Roumains, peuple multimillénaire de l'Europe.
} 
milliards d'impôt sur 5 ans"71. L'échec en Italie détermine Drăgan de poursuivre ses recherches aux Etats-Unis mais le résultat est identique. Certainement les raisons économiques et politiques justifient le refus, mais au-delà de ces aspects techniques, les tendances du cinéma populaire à la fin des années 1970 favorisent d'autres genres comme la science-fiction ou le film d'horreur. De plus, il est certain que le scénario de Gheorghiu n'aurait pas convenu aux producteurs occidentaux à la recherche du spectaculaire et certainement, les producteurs roumains n'auraient pas fait de concessions au divertissement comme dans les années 1960.

L'insuccès de ce projet et les frictions entre Corneliu Leu et la Centrale ont conduit, en juillet 1979, au transfert du film à la maison de production $n^{\circ} 5^{72}$. Dumitru Fernoagă, le directeur et l'un des plus expérimentés producteurs de films historiques, approuve l'entrée en production le 7 août 1979 et accepte le budget de 14 millions de lei $^{73}$. La situation reste inchangée jusqu'en janvier-février 1980 lorsque la Centrale "Româniafilm", probablement après les indications du CCES ou même du Secrétariat du parti ${ }^{74}$, approuve un nouveau devis de 20 millions de $1 \mathrm{e}^{75}$. Les étapes qui ont conduit à la majoration des fonds ne sont pas encore clarifiées, mais il se peut que le résultat anticipé pour un devis de 14 millions de lei fût en dessous des attentes des autorités politiques.

\subsubsection{Quand le réalisateur idéal ne joint pas le film de la décennie}

Le deuxième problème important qui préoccupe la maison de production dès le départ est le choix d'une équipe stable capable de mener à bout un projet en régime d'urgence et au meilleur niveau artistique. Le premier choix a été Sergiu Nicolaescu qui avait déjà fait ses preuves dans les superproductions et qui est devenu depuis une dizaine d'années un personnage très influent dans le milieu cinématographique de l'époque communiste. Sans être forcément un réalisateur "de service", la grande partie de ses œuvres, contrairement à ce qui a été dit ailleurs ${ }^{76}$, reste dans les marges officielles. Grâce à son penchant pour la culture populaire, il contribue de manière plus efficace que les autres cinéastes à la diffusion de l'histoire nationale, de l'histoire du parti, etc. Visiblement, il a été choisi non seulement pour

\footnotetext{
${ }^{71}$ ANF, dossier "Burebista", lettre adressée par Iosif Constantin Drăgan à Corneliu Leu, 26 février 1979.

${ }^{72}$ ANF, dossier "Burebista", Procès-verbal n 257, 3 juillet 1979.

73 ANF, dossier "Burebista", lettre adressée par Dumitru Fernoagă au Centre de Production Cinématographique de Buftea, 7 août 1979.

${ }^{74}$ La note réalisée par la maison de production qui présentait un bilan financier (14 millions de lei) et artistique est censée être arrivée dans le bureau d'Ilie Rădulescu, le secrétaire au niveau de la propagande. ANF, dossier "Burebista", "Notă", non signée, 9 janvier 1980.

${ }^{75}$ ANF, dossier "Burebista", lettre adressée par Dumitru Fernoagă à la Centrale "Româniafilm", 26 février 1980.

${ }^{76}$ Oscar Lapeña Marchena, op.cit., p. 167.
} 
sa maîtrise du tournage des scènes de bataille, mais pour l'attraction de ses films sur le public. Toutefois, son investissement complet dans le projet Burebista s'avère problématique. Au départ, il présente sa version du découpage ${ }^{77}$ (pour un film de 30 millions de lei), prend contact avec le ministère de la Défense Nationale afin d'obtenir 2000 à 3000 militaires pour le tournage $^{78}$ et figure, au moment de l'entrée en production, comme le principal réalisateur. Toutefois, en octobre 1979, Dumitru Fernoagă annonce l'indisponibilité de Nicolaescu et son remplacement avec Gheorghe Vitadinis qui s'était engagé "à réaliser un film selon les paramètres économiques et financiers approuvés par la maison de production $\mathrm{n}^{\circ} 5$ et la Centrale Româniafilm"79.

Les raisons qui ont conduit Nicolaescu hors du projet méritent un regard plus attentif, car il était le réalisateur le plus approprié pour traiter un sujet d'une telle envergure. Le directeur Fernoagă fournit des explications solides pour justifier l'absence de Nicolaescu: l'engagement du réalisateur auprès des autres maisons de production pour la finalisation de ses films précédents et l'inachèvement du découpage pour Burebista au montant de 14 millions de lei. Dans son livre Cartea revoluţiei române decembrie '89, Nicolaescu présente un autre point de vue expliquant les faits par un refus de sa part suite à la lecture du scénario: "j’ai pleuré de rire en voyant comment l'auteur faisait des efforts pour ennoblir les Daces et comment il a amené Burebista à s'exprimer comme à la tribune des congrès du parti, à l'exemple d'un bon diplômé de l'Académie Ştefan Gheorghiu" ${ }^{80}$. Bien que ce soit l'opinion de l'intéressé, la version de Fernoagă nous semble plus plausible. Risible ou pas, le scénario a intéressé Nicolaescu qui, en signant le premier découpage en 1978, accepte la réalisation du film. Quant à ses autres projets en cours, il est vrai qu'il s'était engagé dans cinq films, certains simultanés entre 1978 et 1980. La production de Burebista exigeait un investissement exclusif de sa part, donc son remplacement paraît logique. Toutefois, nous croyons que les limitations financières qui lui sont imposées représentent un obstacle insurmontable. Ses exigences pour les reconstitutions, sans céder sur ses standards, requièrent des sommes très élevées alors que le montant approuvé par la Centrale représente moins de la moitié du budget qu'il exige.

Outre les motivations qui se cachent derrière, le départ de Nicolaescu démontre deux attitudes: d'une part le film n'exerce plus la même séduction sur Nicolaescu qu'avait fait Les

\footnotetext{
${ }^{77}$ ANF, dossier « Burebista », lettre adressée par Corneliu Leu à Miu Dobrescu qui accompagne le découpage du film, 12 décembre 1978.

${ }^{78}$ ANF, dossier « Burebista », lettre adressée par Victor Stănculescu, adjoint du chef de l'État-major à Dumitru Ghişe, 2 août 1979.

${ }^{79}$ ANF, dossier "Burebista", "Minuta" signée par Dumitru Fernoagă et Gheorghe Vitanidis, 19 novembre 1979.

${ }^{80}$ Sergiu NICOLAESCU, Cartea revoluţiei române decembrie '89, Bucureşti: All, 2005, p. 498.
} 
Daces pour le déterminer à renoncer aux autres projets; d'autre part, ni les responsables administratifs, ni les autorités politiques n'ont l'intention ou le pouvoir de l'obliger à se consacrer exclusivement à Burebista .

\subsubsection{Vers une superproduction avec les moyens d'une économie de pénurie}

Après l'insuccès des formalités pour la coproduction, la maison de production et l'équipe de tournage se mobilisent pour réaliser le film par leurs propres moyens. Ils semblent subjugués par une volonté de vaincre tous les obstacles matériels, et la première démarche est la dépense parcimonieuse des matières premières. Le directeur de la maison de production rapporte à ses supérieurs les premières mesures:

A la réalisation du devis, nous avons pris en considération l'utilisation au maximum de toutes les ressources dont dispose le Centre de Production Cinématographique, et en particulier des films similaires réalisés antérieurement ou qui sont en production, comme Les Daces, La Colonne, 1848, Les Montagnes en flammes; l'utilisation des objets qui peuvent être employés dans leur état ou reconditionnés et adaptés à la spécificité de l'époque (costumes, armes) ${ }^{81}$.

La récupération des accessoires des films dont l'action se passe au XIX ${ }^{\mathrm{e}}$ siècle pour les réutiliser dans un film sur l'antiquité prouve l'état extrêmement précaire de la cinématographie. Le budget de 20 millions validé en janvier 1980 est destiné au paiement des professionnels du cinéma impliqués dans la production (réalisateur, scénariste, acteurs, créateurs de costumes, décors, etc) mais il ne couvre pas la totalité des collaborateurs externes. Les conseillers historiques sont les seuls collaborateurs rémunérés régulièrement pour leurs services. Pour les autres domaines, la situation est négociable.

La production des films historiques est dépendante de l'appui d'autres établissements connexes au sujet traité. En Roumanie, l'institution qui s'est fait une tradition de la participation à la réalisation des films est le ministère de la Défense Nationale. L'Armée devient au fil du temps un partenaire constant de la cinématographie. Comme pour les autres productions, le président du CCES entreprend personnellement des démarches auprès du chef de l'État Major ou du ministre de la Défense Nationale dans le but d'obtenir un certain nombre de militaires et l'armement sans frais. En échange, la maison de production devait inscrire sur le générique "film réalisé en collaboration avec le ministère de la Défense Nationale". Dans le cas de Burebista, suivant la tradition déjà instaurée depuis deux décennies, Suzana Gâdea fait appel au général ministre Ion Coman. Suzana Gâdea insiste dans sa lettre sur l'importance de ce film pour l'image de l'armée roumaine : "[Le film] va

\footnotetext{
${ }^{81}$ ANF, dossier "Burebista", "Notă privind vérificarea devizului Burebista", signée Dumitru Fernoagă et Marin Stanciu, 27 février 1980.
} 
mettre en évidence le haut niveau de la civilisation géto-dace et sa grande puissance militaire, estimée par certaines sources historiques à 200000 combattants" $^{\prime 82}$. Nous devons préciser que la référence aux "forces militaires géto-daces" existe uniquement dans le texte adressé à l'Armée, alors qu'elle disparaît dans les autres documents de présentation. Le but de Gâdea est d'intéresser le ministère de la Défense, (en suggérant l'idée que l'histoire de l'armée roumaine était le sujet du film) afin d'obtenir sa participation libre de charges. Le fait que la présidente du CCES est amenée à écrire cette lettre en personne et à utiliser ce genre d'arguments démontre que la participation gratuite de l'armée aux tournages commençait à être problématique.

Non seulement l'armée, mais également les Conseils locaux et le ministère de l'Agriculture et de 1'Industrie Alimentaire, sont contactés pour apporter leur soutien au film. A la différence du ministère de la Défense qui offre ses services gratuitement, le ministère de l'Agriculture demande 200 lei par jour pour chaque cheval prêté ${ }^{83}$. De même, les Conseils locaux ne manifestent pas la même ouverture et disponibilité que pour les films ayant comme sujet des héros populaires. Dans le cas de Tudor, Michel le Brave, Etienne le Grand et Au Carrefour des grandes tempêtes (où le personnage est Avram Iancu), les organisations locales sont plus réceptives et les figurants rassemblés pour le tournage sont plus enthousiastes pour participer. Ni les circonstances économiques ni le sujet traité (Burebista n'est pas un héros issu de la culture populaire, mais reste un personnage découvert par les élites intellectuelles) ne favorisent une solidarité désintéressée.

Les éléments évoqués ci-dessus démontrent que, malgré le rassemblement de forces attendues et espérées, le chemin concret pour arriver à bonne fin passait par un enchevêtrement de relations sociales et professionnelles loin de l'idéal d'unité imaginé. La télévision refuse la collaboration, l'espoir de coproduction s'envole, Corneliu Leu n'est pas à la hauteur de sa mission et est remplacé, Nicolaescu ne finalise pas le travail commencé. De plus, les restrictions budgétaires ont eu souvent un mot trop important dans la prise de décision. L'atmosphère du tournage et la pression ressentie par toute l'équipe du film sont résumées par le réalisateur Gheorghe Vitanidis dans une interview pour la revue Cinema :

A la différence des conditions dans lesquelles ont été réalisés ces films (Les Daces et La Colonne. N.D.A.), nous devons dans quelques mois épuiser toutes les étapes : préparation, tournage, montage, mixage, dans l'espoir de déposer la copie standard au début du mois de juillet. Si l'on prend en compte

\footnotetext{
${ }^{82}$ ANF, dossier "Burebista", lettre adressée par Suzana Gâdea à Ion Coman, 16 janvier 1980.

${ }^{83} \mathrm{ANF}$, dossier «Burebista », lettre adressée par Angelo Miculescu, le ministre de l'Agriculture et de l’Industrie Alimentaire à Suzana Gâdea, 16 février 1980.
} 
les normes habituelles du studio, cela paraît impossible. Je ne sais pas si nous allons y arriver, mais nous allons essayer. Même si cela doit nous coûter du travail intensif, les nerfs, la santé, la vie ${ }^{84}$.

La déclaration de Vitanidis démontre que les producteurs et les réalisateurs de films historiques se sentaient investis d'une mission nationale et patriotique par la transposition en images du passé.

\subsection{Nouvelle conception des Daces}

Le film Burebista s'inscrit dans une nouvelle phase de concevoir le film historique apparue dans la première moitié des années 1970 et qui se manifeste par une diminution des éléments spectaculaires et dramatiques pour accorder plus de place à l'éducation historique et à la fidélité aux sources ${ }^{85}$. Burebista représente certainement le sommet de cette tendance. Cela est visible dans la manière de convoquer et de contractualiser les conseillers historiques. Premièrement, leur nombre est plus important qu'auparavant. Il passe de deux conseillers maximum pour les films des années 1970 à quatre, sans compter les conseillers militaires, pour Burebista: Dumitru Berciu, Constantin Preda, Hadrian Daicoviciu et Florian Georgescu. Deuxièmement, si pour les films précédents la contribution de l'historien se résumait surtout à des rapports de «conformité historique » du scénario, dans Burebista la mission des historiens est plus complexe et ne se réduit pas à un simple rapport, mais à une observation « de terrain ». A l'exception de Georgescu, tous les autres écrivent au minimum un rapport sur le scénario. Troisièmement, si les débats précédents autour du scénario (notamment dans le cas du film Les Daces) rassemblaient plusieurs personnalités du milieu cinématographique et cherchaient un l'équilibre entre la dramatisation et la fidélité aux sources, pour Burebista les débats se portent entre les historiens et le scénariste, soutenu parfois par le réalisateur et le sujet concerne la justesse de la reconstitution.

Le grand changement qu'essaie d'apporter le scénariste dans la représentation de l'antiquité est l'image de la société dace. La décision du parti de célébrer la création de l'État de Burebista est accompagnée d'une description insistant sur "le fleurissement socioéconomique ${ }^{86 "}$ de la société dace, ce que la production tente de respecter. Mais on ne saurait oublier que Mihnea Gheorghiu avait construit cette image dès 1975. Chez Gheorghiu, le fort développement de la civilisation dace est doublé par la conscience politique transcendante de

\footnotetext{
${ }^{84}$ «Burebista. Fierul şi aurul », interview avec Gheorghe Vitanidis réalisée par Roxana Pană, Cinema, $\mathrm{n}^{\circ} 4$, avril 1980, p. 8.

${ }^{85}$ Aurelia VASILE, Le cinéma roumain..., op.cit., p. 322

${ }^{86}$ Nicolae Ceauşescu, România pe drumul construirii societăţii socialiste multilateral dezvoltate, vol. 14, Bucureşti, editura Politică, 1977, p. 317.
} 
Burebista. A cette occasion, Gheorghiu explique sa vision des faits et surtout sa totale confiance dans la vérité de son approche :

Je vous assure de l'authenticité historique absolue de la narration et des personnages qui composent le sujet de mon récit cinématographique et j'invoque pour cela l'autorité des derniers documents révélés par les plus distingués thracologues roumains et étrangers. La civilisation géto-dace se porte étonnamment très bien, en tout cas, mieux que dans les films antérieurs sur le sujet ${ }^{87}$.

Les nouvelles découvertes dont parle Gheorghiu représentent surtout de nouvelles interprétations des anciennes sources, formulées par les défenseurs du courant thracomane. Sur des positions similaires se place Gheorghe Vitanidis qui vient appuyer cette vision des Daces et de Burebista.

\begin{abstract}
Au sujet de la personnalité de Burebista on ne connaît que quelques données morales. Mais on voit qu'il était un grand homme politique, un grand commandant. Le grand géographe et historien grec Strabon disait qu'il avait réussi à élever son peuple et à créer l'un des plus puissants États de l'Europe par trois principes fondamentaux : l'instruction, la modération et le respect des lois. Il existait une instance législative et une autre instance qui vérifiait leur application. Donc, une forme d'organisation supérieure qui dépasse de loin la notion de tribu. Sans engager une polémique avec les films précédents, nous voulons soumettre au public un nouveau point de vue. Nous sommes convaincus que les Daces connaissaient un état avancé de civilisation, ils n'étaient pas un peuple d'analphabètes, mais un peuple qui avait la capacité de communiquer en grec et en latin. Ils connaissaient la philosophie, les sciences astronomiques. Hérodote disait qu'ils étaient les plus braves et les plus droits des Thraces, mais Hérodote avait dit cela 300 ans avant Burebista. Alors, vous pouvez penser que depuis, ils ont évolué encore davantage $[\ldots]^{88}$.
\end{abstract}

Le réalisateur met en évidence non seulement la civilisation supérieure des Daces, mais la supériorité de son film à ceux des années 1960. Pour Vitanidis, Burebista est un moyen de se distinguer de son prédécesseur qui avait décrit les ancêtres comme des tribus barbares.

Afin de soutenir et couvrir scientifiquement ce type de reconstitution, les créateurs de discours historiques du milieu cinématographique font appel à des savants de prestige. Mihnea Gheorghiu invoque 1'autorité scientifique de Mircea Eliade dont la qualité des travaux jouit d'une certaine réputation internationale. ${ }^{89} \mathrm{Il}$ s'est défendu de toute accusation de manipulation des faits historiques en précisant que tous les événements sont authentiques et confirmés par les plus grands historiens de Roumanie ${ }^{90}$. En effet, les idées du scénario ont trouvé appui chez certains des historiens qui ont rédigé les rapports de spécialité.

\footnotetext{
87 "În 1980. Burebista, eroul din filmul Fierul şi aurul", Interview avec Mihnea Gheorghiu réalisée par Adina Darian, Cinema, $\mathrm{n}^{\circ} 1$, janvier 1979, p. 11.

88 "Burebista. Fierul şi aurul", Interview avec Gheorghe Vitanidis réalisée par Roxana Pană, Cinema, $\mathrm{n}^{\circ} 4$, avril 1980 , p. 8

89 "În 1980. Burebista, eroul din filmul Fierul şi aurul", Interview avec Mihnea Gheorghiu réalisée par Adina Darian, Cinema, $\mathrm{n}^{\circ} 1$, janvier 1979, p. 11.

${ }^{90}$ Archives personnelles, entretien avec Mihnea Gheorghiu réalisé en août 2005.
} 


\section{Conclusions}

Les films roumains traitant de l'antiquité témoignent par leur mode de production des transformations d'ordre politique, idéologique et économique qui se produisent dans le milieu cinématographique au cours de deux dernières décennies du régime communiste. Réalisé à des périodes différentes, le retour thématique vers les origines nationales relève des besoins différents: affirmation identitaire durant les années 1960 et exaltation de l'exceptionnalité des ancêtres dans les années 1980. L'approche de la production des films démontre une intention visible chez les participants (cinéastes, bureaucrates, autorités politiques) de rendre service à la nation par le biais du film. Dans les années 1960, grâce à l'ouverture internationale du Studio "Bucureşti" pour les coproductions, la représentation des Daces en tant que pères fondateurs des Roumains pouvait transgresser les frontières. Toutefois, cette exposition internationale a eu son propre coût qui a pris la forme de concessions au marché et au goût du grand public. Dix ans après, lors de la production du film Burebista, l'image des Daces devait transmettre le haut niveau de la civilisation antique, mais la relation avec l'étranger avait pris une autre direction: le système de coproduction avait disparu et les compagnies étrangères avaient perdu l'intérêt pour ce genre de sujet. Dans ce cas, le film se referme sur soi: il est produit par la maison de production roumaine, sous les auspices du Parti Communiste Roumain pour parler aux spectateurs roumains de leur gloire passée.

Les conditions de production et la mobilisation humaine autour de ces projets démontrent le véritable engouement que les sujets nationaux représentaient pour le milieu cinématographique. Si dans les années 1960, les participants aux films sur les Daces semblaient être motivés par une mission patriotique transcendante, les difficultés financières des années 1980 et probablement l'origine politique du projet, a déstabilisé la ferveur générale. Néanmoins, les professionnels du studio et le ministère se sont engagés complètement dans l'accomplissement de leur tâche d'importance nationale. 